\title{
Fractal Structure of Zeros in Hierarchical Models
}

\author{
B. Derrida, ${ }^{1}$ L. De Seze, ${ }^{1}$ and C. Itzykson ${ }^{1}$
}

Received June 28, 1983

We consider an Ising and a $q$-state Potts model on a diamond hierarchical lattice. We give pictures of the distribution of zeros of the partition function in the complex plane of temperatures for several choices of $q$. These zeros are just the Julia set corresponding to the renormalization group transformation.

KEY WORDS: Zeros; partition function; hierarchical models; mapping; Julia set.

\section{INTRODUCTION}

Recently, hierarchical lattices have motivated a lot of work. ${ }^{(1-9)}$ These lattices are constructed in such a way that the bond moving renormalization approximation due to Migdal ${ }^{(10)}$ and Kadanoff ${ }^{(11)}$ becomes exact. In other words, using the Migdal-Kadanoff renormalization, one can solve exactly a variety of models of statistical mechanics on these hierarchical lattices. Although these solutions are usually rather simple, the critical behaviors and the phase diagram may be very rich. In particular, the critical exponents are nontrivial. Moreover in some cases with frustration, the renormalization group transformation may have chaotic trajectories. ${ }^{(5-9)}$ In all cases, a large amount of information, like the exact values of the exponents or the phase diagram, can be extracted from the knowledge of the renormalization group transformation. When the rule of construction of the hierarchical lattice is simple enough (see Refs. 2 and 4 for a variety of such models) the renormalization group transformation can be written as a simple rational map in the space of the parameters which define the model.

\footnotetext{
${ }^{1}$ Commissariat a l'Energie Atomique, Institut de Recherche Fondamentale, Departement de Physique Generale, Services de Physique Theorique et de Physique du Solide, Cen-Saclay91191 Gif-sur-Yvette Cedex-France.
} 
The rational transformations have been studied for a long time $e^{(12,13)}$ with a renewed interest ${ }^{(14-18)}$ recently in the theory of bifurcations. A particular interest was concentrated on the Julia sets in the complex plane ${ }^{(17-19)}$ For a rational mapping, the Julia set is the set of points which do not belong to any basin of attraction. As shown in Refs. 17 and 18, even for very simple rational transformations, the Julia sets have usually a fractal structure which is quite decorative.

The purpose of the present work is to obtain the Julia sets corresponding to the renormalization group transformations which appear in the study of hierarchical models. The Julia set of a renormalization transformation is nothing but the limiting set of all the zeros in the complex plane of the partition function. For hierarchical models, one can obtain the complete structure of the zeros of the partition function, a problem which has a long history since the famous Lee and Yang ${ }^{(20)}$ theorem. In the complex plane of temperatures, ${ }^{(21)}$ except in a few exactly solvable cases, the location of these zeros is a hard prolem. ${ }^{(22,23)}$ One does not know if they are concentrated on curves, on sectors of the plane, or on more complicated regions. Therefore, it is interesting to see what are these regions in the case of hierarchical lattices. One should keep in mind that sometimes, features found on hierarchical lattices are very unusual ${ }^{(3)}$ and so it is very possible that the structures which are shown below do not persist on regular lattices.

The paper is organized as follows. In Section 2, we recall the construction of a simple hierarchical lattice: the diamond one. For $q$-state Potts spins, we write the renormalization transformation and we obtain the recursion relation which gives the partition function. In Section 3, we explain why the Julia set of the renormalization transformation is the set of zeros of the partition function. In Section 4 we present pictures of this Julia set for several values of $q$.

\section{THE DIAMOND HIERARCHICAL MODEL}

In this paper, we shall restrict ourselves to one of the simplest hierarchical lattice: the diamond one. A construction of the diamond hierarchical lattice is schematized in Fig. 1. ${ }^{(3)}$ One starts with one bond in Fig. 1a and the first step is to consider that the bond of Fig. 1a is an effective bond which represents the effect of the four bonds of Fig. 1b. So going from 1a to $1 \mathrm{~b}$, one has replaced one bond by a set of four bonds. Similarly, one can go from $1 \mathrm{~b}$ to $1 \mathrm{c}$ by replacing each bond of Fig. $1 \mathrm{~b}$ by a set of four bonds. If one iterates this procedure an infinite number of times, one obtains the diamond hierarchical lattice.

If we put a $q$-state Potts model on this hierarchical lattice, the recursive 


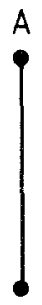

B

(a)

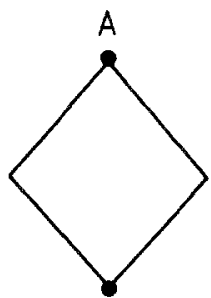

B

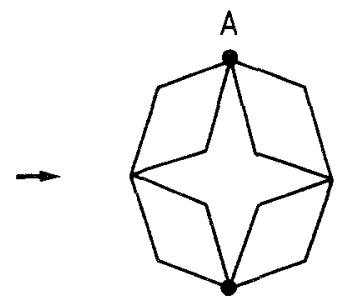

B

(c)

Fig. 1. The recursive construction of the diamond hierarchical model.

construction allows us to calculate the free energy exactly. ${ }^{(24,7)}$ The Hamiltonian of a $q$-state Potts model is

$$
\mathscr{H}=-\sum_{\langle i j\rangle} J \delta_{\sigma_{i} \sigma_{j}}
$$

where the sum runs over all pairs of nearest neighbors on the lattice, the $\sigma_{i}$ can take $q$ possible values $\left(\sigma_{i}=1,2, \ldots, q\right)$ and $\delta$ is the usual Kronecker symbol. To simplify the notations in the remainder of the paper, everything will be expressed as function of $y$ defined by

$$
y=\exp \beta J
$$

where $\beta$ is as usual the inverse temperature. The properties of the free energy or of the partition function in the complex plane of $y$, can of course, be easily related to what they are in the complex plane of $\beta$.

Let us consider that Fig. 1a represents the first step in the construction of the lattice, Fig. $1 \mathrm{~b}$ represents the second step, Fig. 1c represents the third step, and so on. One can calculate the partition function $Z_{n}(y)$ of a Potts model on the finite lattice constructed after the $n$th step if one knows the partition function $Z_{n-1}\left(y^{\prime}\right)$ for the finite lattice constructed after $n-1$ steps. This relation can be easily established by taking the trace over all the spins which are connected to only two other spins in the finite lattice constructed after $n$ steps:

$$
Z_{n}(y)=Z_{n-1}\left(y^{\prime}\right)[A(y)]^{2.4^{n-2}}
$$

where the function $A(y)$ is given by

$$
A(y)=2 y+q-2
$$


and $y^{\prime}$ is the image of $y$ by a transformation $T$ :

$$
y^{\prime}=T(y)=\left(\frac{y^{2}+q-1}{2 y+q-2}\right)^{2}
$$

The transformation $T$ is the renormalization transformation of the model. The advantage of hierarchical lattices is that one can find $T$ exactly. By iterating Eq. (3) $n-1$ times, one can calculate $Z_{n}(y)$ for any value of $y$ since $Z_{1}(y)$ is known:

$$
Z_{1}(y)=q(y+q-1)
$$

Therefore the partition, the free energy, and its derivatives can be easily calculated from (3).

\section{THE ZEROS OF THE PARTITION FUNCTION}

Since on the finite lattice constructed after $n$ steps there are $4^{n-1}$ bonds, the partition function $Z_{n}(y)$ is a polynomial of degree $4^{n-1}$ in $y$. The term of highest degree is $q y^{4^{n-1}}$. Therefore, one can write $Z_{n}(y)$ in the following way:

$$
Z_{n}(y)=q \prod_{i=1}^{4^{n-1}}\left(y-x_{i}\right)
$$

where the $x_{i}$ are by definition the zeros of $Z_{n}(y)$.

If we denote by $\tilde{x}_{i}$ with $1 \leqslant i \leqslant 4^{n-2}$ the zeros of $Z_{n-1}(y)$, one sees that Eqs. (3), (4), and (5) give a relation between the $x_{i}$ and the $\tilde{x}_{i}$ :

$$
q \prod_{i=1}^{4^{n-1}}\left(y-x_{i}\right)=q\left\{\prod_{i=1}^{4^{n-2}}\left[\left(\frac{y^{2}+q-1}{2 y+q-2}\right)^{2}-\tilde{x}_{i}\right]\right\}(2 y+q-2)^{2 \cdot 4^{n-2}}
$$

which can be rewritten as

$$
\prod_{i=1}^{4^{n-1}}\left(y-x_{i}\right)=\prod_{i=1}^{4^{n-2}}\left[\left(y^{2}+q-1\right)^{2}-\tilde{x}_{i}(2 y+q-2)^{2}\right]
$$

Let us look at one factor (for example, the one with $\tilde{x}_{1}$ ) in the product at the right-hand side of (9):

$$
P(y)=\left(y^{2}+q-1\right)^{2}-\tilde{x}_{1}(2 y+q-2)^{2}
$$

$P(y)$ is a polynomial of degree 4 whose four roots are the four preimages of 
$\tilde{x}_{1}$ by $T$. This is due to the following equivalence:

$$
P(y)=0 \Leftrightarrow T(y)=\tilde{x}_{1}
$$

This fact, together with (9), proves that if one knows the $4^{n-2}$ zeros $\tilde{x}_{i}$ of $Z_{n-1}(y)$, then one knows the $4^{n-1}$ zeros $x_{i}$ of $Z_{n}(y)$. Thus $x_{i}$ are just the preimages of the $\tilde{x}_{i}$ by the renormalization transformation $T$. Therefore, since one knows $Z_{1}(y)$, which has only one zero at $y=1-q$, one knows all the zeros of all the $Z_{n}(y)$. The zeros of $Z_{n}(y)$ are just the $4^{n-1}$ preimages of $1-q$ by the $(n-1)$ th iterate of the transformation $T$.

In the thermodynamic limit $n \rightarrow \infty$, this set of preimages is exactly what is called the Julia set of the transformation $T .^{(13,17-19)}$ Therefore we have shown here that the zeros of the partition function $Z_{n}(y)$ give in the thermodynamic limit the Julia set of the renormalization transformation.

\section{THE PICTURES}

We have seen that $Z_{n}(y)$ has $4^{n-1}$ zeros. For large $n$ there is no chance to calculate all of them. We have used a Monte Carlo procedure to obtain pictures of the set of zeros of $Z_{n}(y)$ in the limit $n \rightarrow \infty$. The method is very simple. We start with any point $y_{0}$. It has four preimages by the transformation $T$. We choose one of them $y_{1}$ at random. Then we calculate its four preimages and we choose again one of them $y_{2}$ at random and so on. After a few iterations, where at each step we choose randomly one of the four preimages of the previous point, we have a point which is extremely close from the Julia set. Then we continue the calculation but we can plot the points since they are on the Julia set (within the numerical accuracy of the computer).

In other words, we calculate the successive preimages of a given point $y_{0}$. But as there are at each step four preimages, we choose one of them at random. We plot these preimages only after a few steps in order to eliminate the first ones which are not close enough to the Julia set. One should notice that it is not necessary to choose $y_{0}=1-q$ because the Julia set is independent of the starting point $y_{0}$.

Figure 2 gives the picture obtained by plotting 20,000 points of the Julia set. This figure corresponds to the Ising model $(q=2)$. One sees clearly the well-known fractal structure of the Julia set and therefore we conclude that the partition function of a hierarchical model has usually a fractal set of zeros in the complex plane of temperatures. In Fig. 2, we did the calculation by choosing at each step one of the four preimages with probability $1 / 4$. Therefore, the points are distributed on Fig. 2 according to the true density of zeros. However, even with 20,000 points, one does not see clearly the envelope and the whole support of the Julia set. 


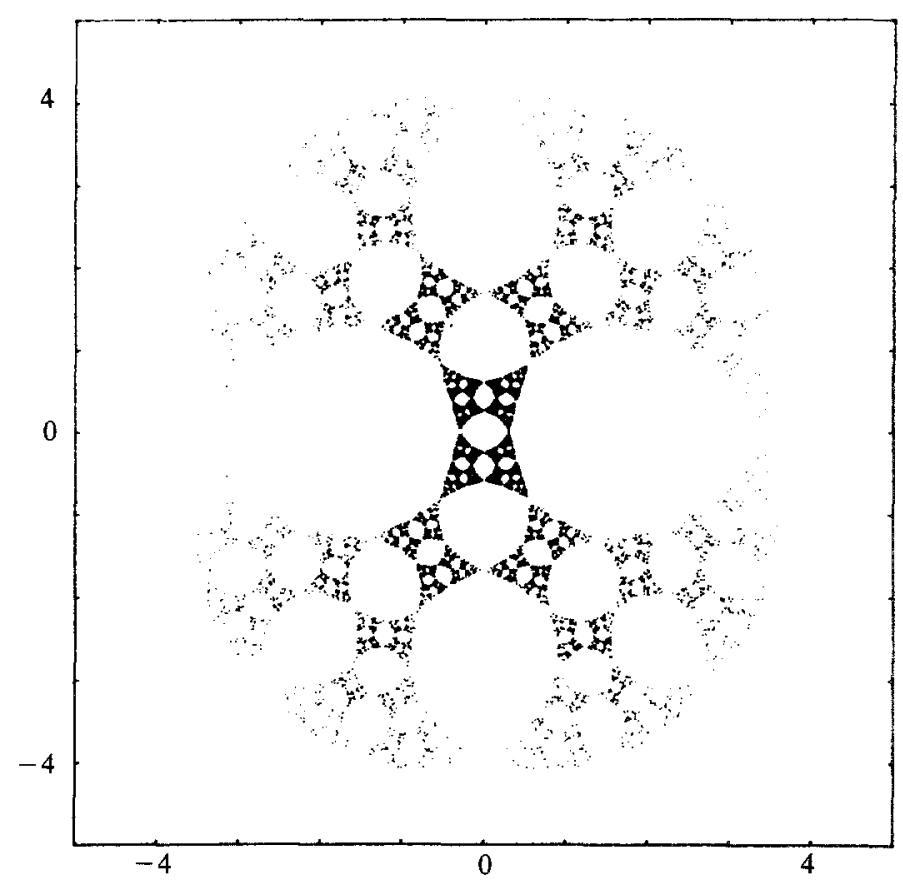

Fig. 2. 20,000 points of the Julia set of the renormalization transformation obtained by the Monte Carlo procedure explained in the text. These points are zeros of the partition function in the Ising case $(q=2)$.

To see better the support of the Julia set, we decided to make the same calculation but with a bias in order to have more points in the low-density regions. In other words, we have chosen with a higher probability the preimages which are in low-density regions. Doing so we have obtained Fig. 3 for the Ising case $(q=2)$. It is the same as in Fig. 2 but one sees more clearly the support of the Julia set. One should not forget that Fig. 3 gives the support of the set but, because of the bias, the density of points in Fig. 3 does not represent the true density of zeros of $Z_{n}(y)$ in the limit $n \rightarrow \infty$. The physical region is the positive part of the real axis: $y>1$ corresponds to the ferromagnetic model, whereas $0<y<1$ corresponds to the antiferromagnetic model. In the Ising case, the symmetry between the ferromagnetic and the antiferromagnetic model makes the figure invariant in the change $y \rightarrow 1 / y$. One sees clearly in Fig. 3 the ferromagnetic transition at $y_{F} \simeq 3.383$ and the antiferromagnetic transition at $y_{A} \simeq 0.2956$.

Figures 4-7 represent again as in Fig. 3 the support of the Julia set, i.e., the support of the zeros of the partition function $Z_{n}(y)$ for other values of $q: q=1.5,2.5,3$, and 4 . One sees also that, for these other values of $q$, 


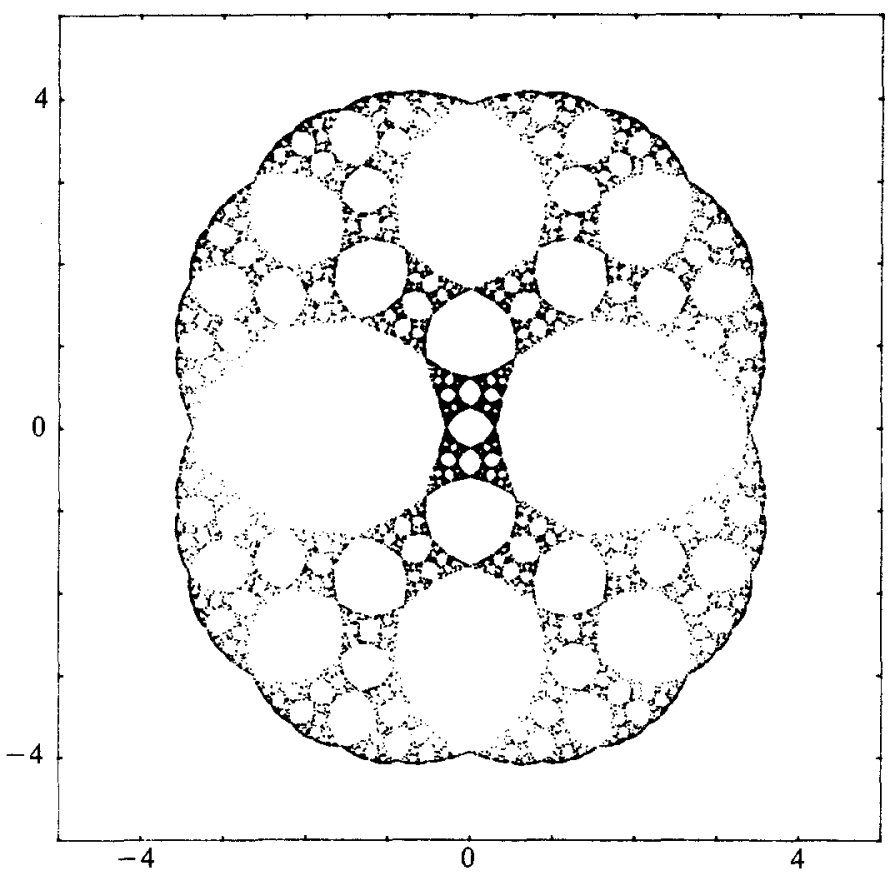

Fig. 3. The same as Fig. 2 but with the bias in the Monte Carlo calculation to see better the support of the set of zeros.

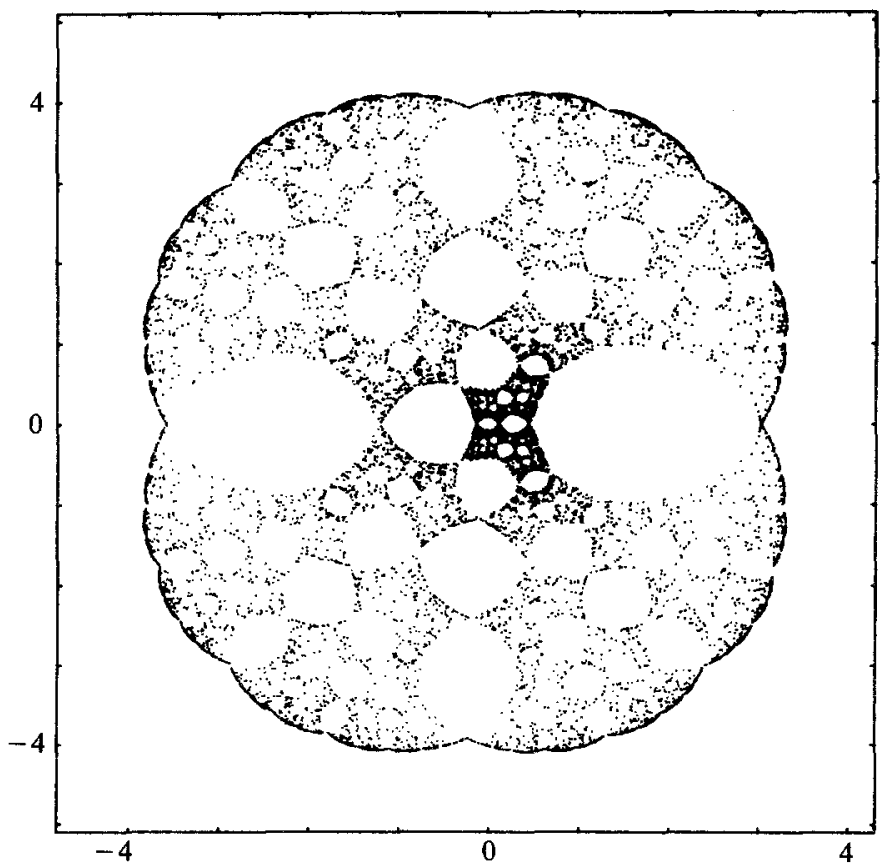

Fig. 4. The same as Fig. 3 for $q=1.5$. 


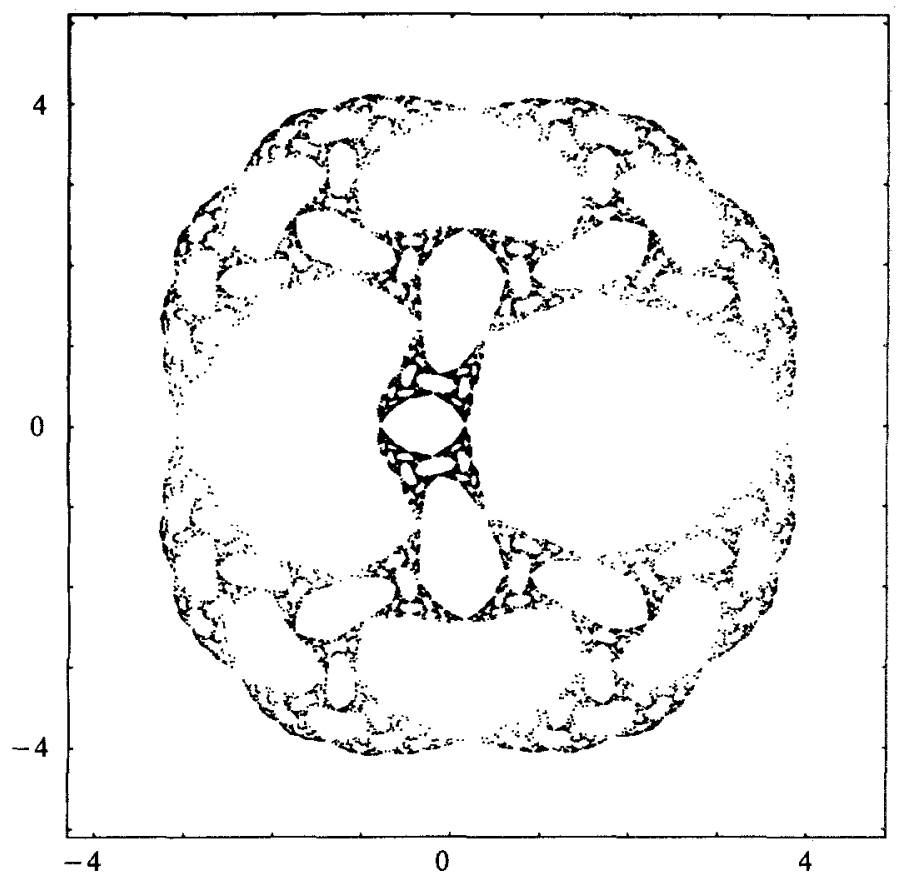

Fig. 5. The same as Fig. 3 for $q=2.5$.

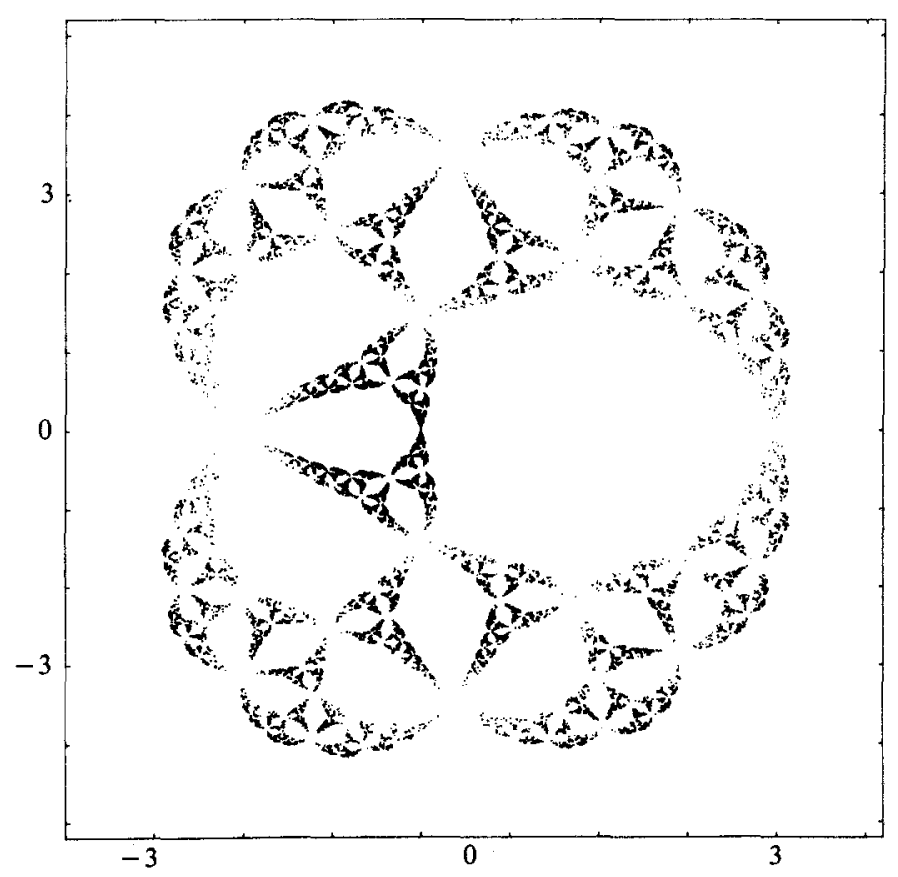

Fig. 6. The same as Fig. 3 for $q=3$. 


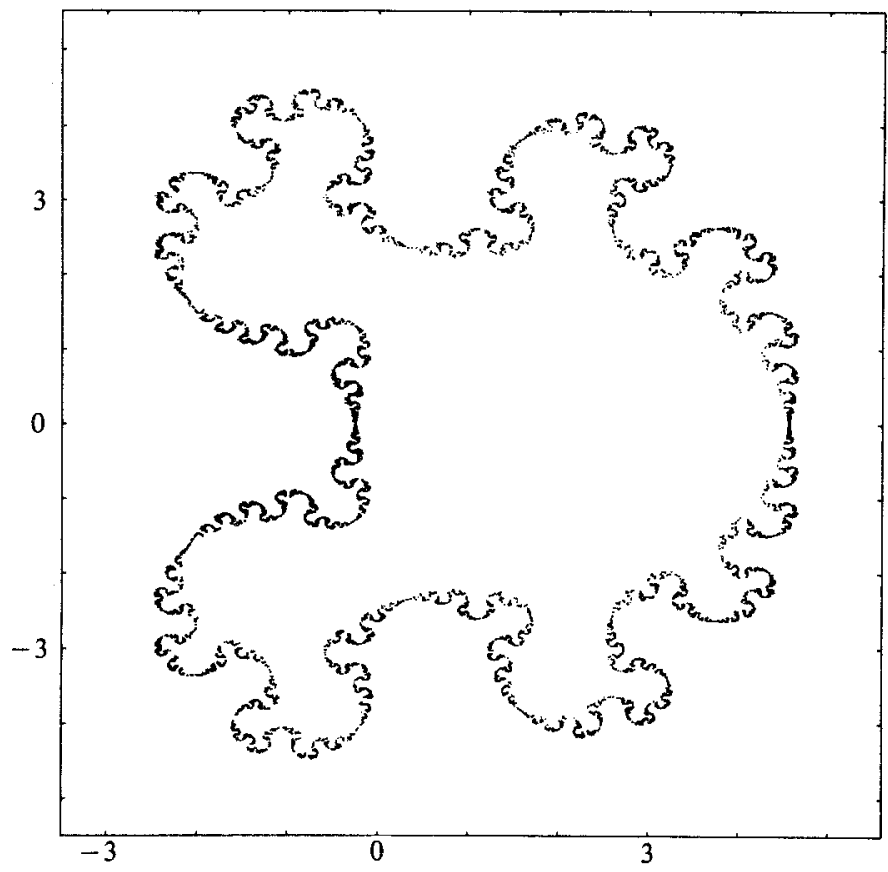

Fig. 7. The same as Fig. 3 for $q=4$.

the Julia set has a fractal structure. One can easily verify that the antiferromagnetic transition disappears for $q>3$ since $y_{A}=0$ for $q=3$.

\section{CONCLUSION}

We have shown in this paper that for a family of hierarchical models, the Julia set of the renormalization transformation gives the zeros of the partition function in the thermodynamic limit. The Julia set contains all the unstable periodic points of the renormalization transformation. In cases where, owing to frustration, ${ }^{(5-9)}$ the renormalization group transformation has a lot of unstable periodic points ${ }^{(7)}$ on the real axis, the Julia set contains much more points on the real axis giving rise to much more singularities of the free energy in the physical region.

As observed by several authors ${ }^{(7,24)}$ the singular behavior of the free energy near a critical fixed point $y_{c}$ is in principle modulated because of the discreteness of the renormalization transformation:

$$
\text { singular part }\left[\lim _{n \rightarrow \infty} \frac{1}{4^{n-1}} \log Z_{n}(y)\right] \sim\left|y-y_{c}\right|^{2-\alpha} H\left\{\frac{\log \left|y-y_{c}\right|}{\log \left[T^{\prime}\left(y_{c}\right)\right]}\right\}
$$


where $H$ is a periodic function of period 1 ,

$$
H(z)=H(z+1)
$$

and $2-\alpha=\log 4 / \log \left[T^{\prime}\left(y_{c}\right)\right]$.

In the Ising case, we did observe numerically these modulations near the ferromagnetic critical point but these modulations are very small: they are of order $10^{-5}$ compared with the mean value of $H$. This means that for hierarchical models the usual idea of a critical amplitude has to be replaced by a periodic function of $\log \left(y-y_{c}\right)$. Encouraged by a recent work ${ }^{(23)}$ which relates the critical amplitudes to the angle of the density of zeros near a critical point, we tried to relate the angles of the Julia set with the real axis which can be seen in Fig. 3 to the values of $H$ that we calculated numerically. In such a naive form our attempt was unsuccessful. Therefore we think that it would be interesting to understand what are the physical quantities which could be related to these angles, for instance the relative amplitudes of modulations.

Lastly, the most important question is to know whether the fractal structure of the set of zeros which seems to be common to almost all the hierarchical models persists at least on some regular lattices. For the Ising model in $d=2$, this is of course not true. ${ }^{(21)}$ However, one can imagine that in cases where the critical behaviors are more complicated than in the two-dimensional Ising model, parts of the set of zeros have a fractal structure. We think that one way to answer this question would be to construct a sequence of hierarchical models which approximate a regular lattice better and better and to see if parts of the fractal structure persist when the hierarchical models become closer and closer to the regular lattice.

\section{ACKNOWLEDGMENTS}

We would like to thank D. Bessis, J. Geronimo, R. B. Griffiths, and P. Moussa for discussions.

\section{REFERENCES}

1. A. N. Berker and S. Ostlund, J. Phys. C12:4961 (1979).

2. M. Kaufman and R. B. Griffiths, Phys. Rev. B 24:496 (1981).

3. M. Kaufman and R. B. Griffiths, J. Phys. A15:L239 (1982).

4. R. B. Griffiths and M. Kaufman, Phys. Rev. B 26:5022 (1982).

5. S. McKay, A. N. Berker, and S. Kirkpatrick, Phys. Rev. Lett. 48:767 (1982).

6. N. M. Svrakic, J. Kertész, and W. Selke, J. Phys. A15:L427 (1982).

7. B. Derrida, J. P. Eckmann, and A. Erzan, J. Phys. A16:893 (1983). 
8. J. R. Melrose, J. Phys. A16:1041 (1983).

9. A. Erzan, Phys. Lett. A 93A:237 (1983).

10. A. A. Migdal, Sov. Phys. JETP 42:743 (1976).

11. L. P. Kadanoff, Ann. Phys. (N.Y.) 100:359 (1976).

12. P. Fatou, Bull. Math. France 47:161 (1919); 48:33, 208 (1920).

13. G. Julia, J. Math. Pures Appl. 4:47 (1918).

14. M. J. Feigenbaum, J. Stat. Phys. 19:339 (1978).

15. B. Derrida, A. Gervois, and Y. Pomeau, J. Phys. A12:269 (1979).

16. P. Collet and J. P. Eckmann, Iterated Maps on the Interval as Dynamical Systems (Birkhäuser, Boston, 1980), and references therein.

17. B. Mandelbrot, Ann. N.Y. Acad. Sci. 357:249 (1980).

18. A. Douady, Séminaire Bourbaki No. 599 (1982-1983).

19. D. Bessis, J. Geronimo, and P. Moussa, to appear in J. Stat. Phys.

20. C. N. Yang and T. D. Lee, Phys. Rev. 87:404 (1952); T. D. Lee and C. N. Yang, Phys. Rev. 87:410 (1952).

21. M. E. Fisher, in Lecturers in Theoretical Physics, Vol. VIIC (University of Colorado Press, Boulder, 1965), p. 1.

22. R. B. Pearson, Phys. Rev. B 26:6285 (1982).

23. C. Itzykson, R. B. Pearson, and J. B. Zuber, Nucl. Phys. B, 220(FS8):415 (1983).

24. Th. Niemeijer and J. M. J. van Leeuwen, in Phase Transitions and Critical Phenomena, Vol. 6, C. Domb and M. S. Green, eds. (Academic Press, New York, 1976), p. 425. 